University of New Orleans

ScholarWorks@UNO

\title{
Tilted bilayer membranes as simple transmission quarter-wave retardation plates
}

\author{
R. M.A. Azzam \\ University of New Orleans, razzam@uno.edu \\ Fadi A. Mahmoud
}

Follow this and additional works at: https://scholarworks.uno.edu/ee_facpubs

Part of the Electrical and Electronics Commons, and the Optics Commons

\section{Recommended Citation}

R. M. A. Azzam and Fadi A. Mahmoud, "Tilted bilayer membranes as simple transmission quarter-wave retardation plates," J. Opt. Soc. Am. A 18, 421-425 (2001)

This Article is brought to you for free and open access by the Department of Electrical Engineering at ScholarWorks@UNO. It has been accepted for inclusion in Electrical Engineering Faculty Publications by an authorized administrator of ScholarWorks@UNO. For more information, please contact scholarworks@uno.edu. 


\title{
Tilted bilayer membranes as simple transmission quarter-wave retardation plates
}

\author{
R. M. A. Azzam \\ Department of Electrical Engineering, University of New Orleans, Lakefront, New Orleans, Louisiana 70148
}

Fadi A. Mahmoud

Adaptec, Inc., 691 South Milpitas Boulevard, Milpitas, California 95035

Received June 5, 2000; accepted July 26, 2000; revised manuscript received August 22, 2000

\begin{abstract}
A tilted bilayer membrane, which consists of two thin films of transparent optically isotropic materials of different refractive indices, can function as a transmission quarter-wave retarder (QWR) at a high angle of incidence. A specific design using a cryolite-Si membrane in the infrared is presented, and its tolerances to small shifts of wavelength, incidence angle, and film thickness errors are discussed. Some designs provide a dual QWR in transmission and reflection. Such devices provide simple linear-to-circular (and circular-to-linear) polarization transformers. Bilayer eighth-wave retarders without diattenuation are also introduced. (C) 2001 Optical Society of America

OCIS codes: $120.2130,230.0230,240.0310,260.5430$.
\end{abstract}

\section{INTRODUCTION}

Wave retarders are versatile optical devices for the control and the analysis of polarized light with numerous applications. The desired differential phase shift between two orthogonal linear polarization components of incident light is often introduced by using linear birefringence in a crystalline plate or total internal reflection. ${ }^{1}$

Coherent multiple-beam interference in a tilted parallel-plane thin dielectric slab (membrane or pellicle) leads to a differential transmission phase shift $\Delta_{t}$ between the parallel $(p)$ and perpendicular $(s)$ polarizations; hence such a slab of an optically isotropic material (Fig. 1) acts as the simplest possible wave retardation plate. $^{2}$ The retardance $\Delta_{t}$ vanishes at normal incidence, and hence the effect can be considered to arise from form birefringence associated with the tilt of the slab. The upper bound on $\Delta_{t}$ is given by ${ }^{3}$

$$
\Delta_{t \max }=\arctan \{[N-(1 / N)] / 2\},
$$

where $N=N_{1} / N_{0}$ is the refractive index of the slab relative to that of the surrounding medium (usually air or vacuum, in which case $N_{0}=1$ ). The maximum retardance is attainable in the limit of grazing incidence, and, according to Eq. (1), its value is $<90^{\circ}$, so that a single homogeneous slab cannot perform as a quarter-wave retarder (QWR). On the other hand, an eighth-wave retarder (EWR) is readily achieved with a pellicle of refractive index $N>1.4966$ at an angle of incidence that decreases as $N$ increases.

In this paper it is shown that this basic limitation of the homogeneous thin slab is lifted if the slab is coated with a transparent thin film of a different refractive index. Interference in such a bilayer membrane gives a QWR, albeit at a high angle of incidence. A specific infrared (IR) QWR design using a cryolite $\left(\mathrm{Na}_{3} \mathrm{AlF}_{6}\right)$ - $\mathrm{Si}$ pellicle is presented. A dual QWR in transmission and reflection is also realizable with certain bilayer membranes. The coated pellicle QWR is a simple (noncrystalline) device that introduces no significant lateral or angular deviation in the transmitted beam. It can be mounted in a rotary stage such that its surface normal precesses in a cone around the incident light beam as an axis. Thus a QWR of variable fast-axis azimuth is obtained. Reflection phase retarders that use periodic or quasiperiodic stacks of many layers have been described by several authors. ${ }^{4-6}$

\section{BASIC RELATIONS}

The change in the state of polarization of light upon transmission through a bilayer membrane (Fig. 2) is determined by the ratio of complex-amplitude transmission coefficients for the $p$ and $s$ polarizations:

$$
\rho_{t}=T_{p} / T_{s}=\left(\tan \psi_{t}\right) \exp \left(j \Delta_{t}\right) .
$$

Explicit expressions for the transmission (and reflection) coefficients of a bilayer (on a substrate) are available substitution of these expressions into Eq. (2) gives

$$
\begin{gathered}
\rho_{t}=a\left(1+b X_{1}+c X_{2}+d X_{1} X_{2}\right) / \\
\left(1+e X_{1}+f X_{2}+g X_{1} X_{2}\right)
\end{gathered}
$$

in which

$$
\begin{aligned}
& a=\left(t_{01 p} / t_{01 s}\right)\left(t_{12 p} / t_{12 s}\right)\left(t_{20 p} / t_{20 s}\right), \\
& b=r_{01 s} r_{12 s}, \quad e=r_{01 p} r_{12 p}, \\
& c=r_{12 s} r_{20 s}, \quad f=r_{12 p} r_{20 p}, \\
& d=r_{01 s} r_{20 s}, \quad g=r_{01 p} r_{20 p} .
\end{aligned}
$$




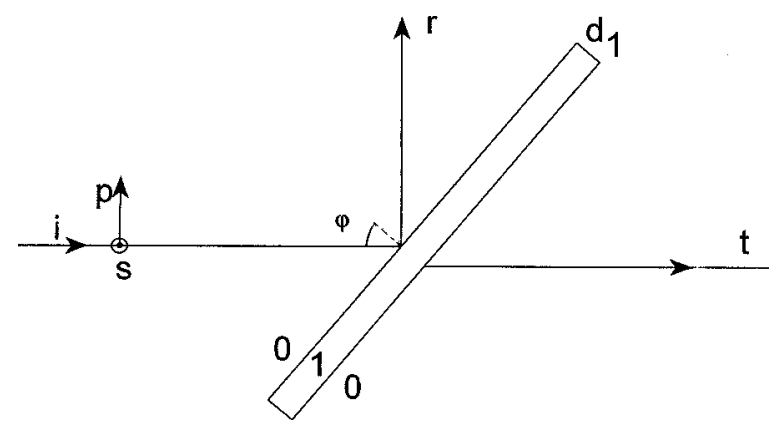

Fig. 1. Reflection and transmission of light by a tilted transparent thin slab of an optically isotropic material (medium 1 of thickness $d_{1}$ ) in a transparent ambient (medium 0 ). $\quad p$ and $s$ are the linear polarizations parallel and perpendicular to the plane of incidence, respectively, and $\phi$ is the angle of incidence.

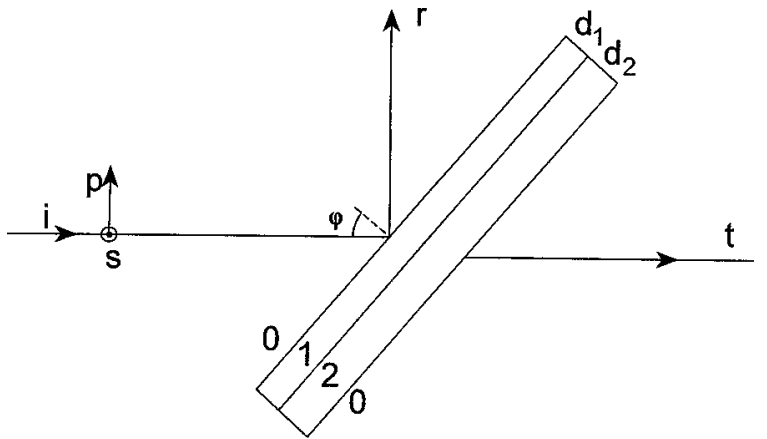

Fig. 2. Reflection and transmission of light by a tilted bilayer membrane of two optically isotropic thin films (media 1 and 2 of thicknesses $d_{1}$ and $d_{2}$, respectively) in a transparent ambient (medium 0). $\quad p$ and $s$ are the linear polarizations parallel and perpendicular to the plane of incidence, respectively, and $\phi$ is the angle of incidence.

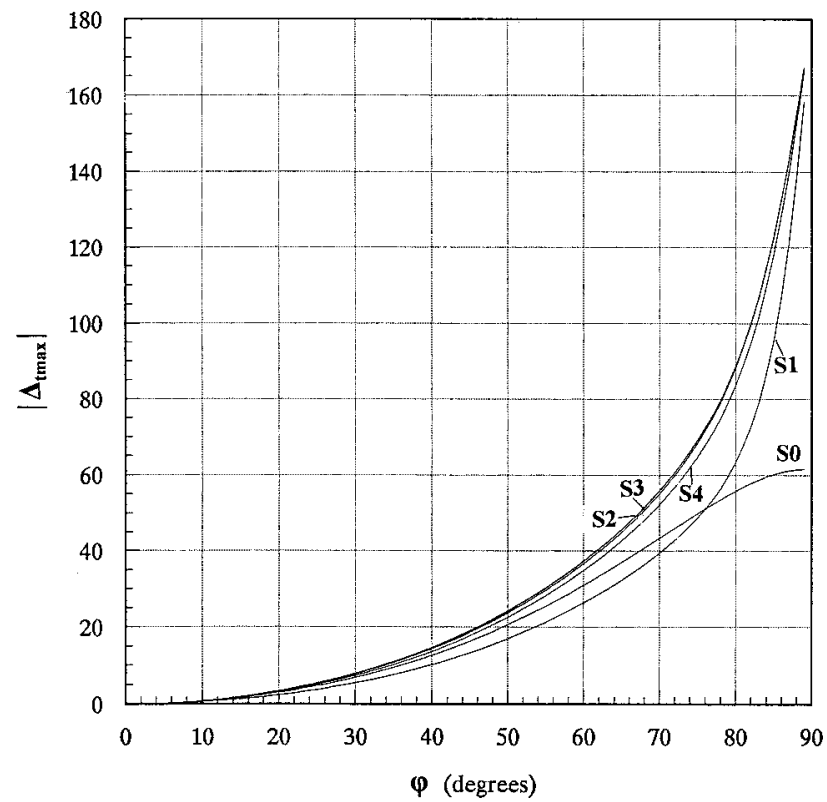

Fig. 3. Maximum differential phase shift in light transmission through a bilayer, $\left|\Delta_{t \max }\right|$, versus angle of incidence $\varphi$ for five material systems $\mathrm{S} 0, \mathrm{~S} 1, \mathrm{~S} 2, \mathrm{~S} 3$, and $\mathrm{S} 4$, which correspond to $\left(N_{1}, N_{2}\right)=(4,4),(1.38,2.35),(1.35,4),(1.25,4)$, and $(1.35$, $3.42)$, respectively. $r_{i j \nu}$ and $t_{i j \nu}$ are the Fresnel reflection and transmission coefficients of the $i j$ interface for the $\nu$ polarization $(\nu=p, s)$, and

$$
X_{i}=\exp \left(-j 2 \pi \zeta_{i}\right), \quad i=1,2,
$$

where $\zeta_{i}$ is the normalized thickness of the $i$ th film, i.e.,

$$
\zeta_{i}=d_{i} / D_{i}, \quad i=1,2
$$

and

$$
D_{i}=(\lambda / 2)\left(N_{i}^{2}-\sin ^{2} \phi\right)^{-1 / 2}, \quad i=1,2,
$$

is the corresponding film thickness period, $\lambda$ is the wavelength of the incident light, $\varphi$ is the angle of incidence, $N_{i}$ is the refractive index of the $i$ th film, and light is assumed to be incident from air or vacuum $\left(N_{0}=1\right)$.

\section{CONSIDERATION OF SEVERAL MATERIAL SYSTEMS}

For given refractive indices $\left(N_{1}, N_{2}\right)$ of the two films of the bilayer, the normalized film thicknesses $\left(\zeta_{1}, \zeta_{2}\right)$ are iterated on in search of the maximum transmission differential phase shift $\Delta_{t \max }$ at a given angle of incidence $\varphi$ by using Eqs. (2)-(5). Figure 3 is a plot of $\left|\Delta_{t \max }\right|$ versus $\varphi$ for five material systems $\mathrm{S} 0, \mathrm{~S} 1, \mathrm{~S} 2, \mathrm{~S} 3$, and $\mathrm{S} 4$ which correspond to $\left(N_{1}, N_{2}\right)=(4,4),(1.38,2.35)$, $(1.35,4)$, $(1.25,4)$, and $(1.35,3.42)$, respectively. S0 reduces to the case of a single homogeneous film of Ge. S1 represents a $\mathrm{MgF}_{2}-\mathrm{ZnS}$ system in the visible, and $\mathrm{S} 2-\mathrm{S} 4$ represent a low-index (fluoride) film on a high-index $\mathrm{Si}$ or $\mathrm{Ge}$ layer in the IR. ${ }^{8}$ The following conclusions are readily drawn from Fig. 3.

1. The upper bound on $\left|\Delta_{t \max }\right|$ for the S0 reference case (the homogeneous Ge layer) is $61.93^{\circ}$, in agreement with Eq. (1).

2. For a bilayer of two films of different refractive indices, the upper bound on $\left|\Delta_{t \max }\right|$ is $180^{\circ}$ at grazing incidence $\left(\phi=90^{\circ}\right)$.

3. For a bilayer of two films of different refractive indices, $\left|\Delta_{t}\right|=90^{\circ}$ is possible at a high angle of incidence $\phi$ $\left(>80^{\circ}\right)$. Therefore a bilayer membrane can be used as a QWR in transmission. To stay as far from grazing incidence as possible, the refractive-index contrast between the two films of the bilayer should be as high as possible, which is attained in the IR spectral region.

\section{CRYOLITE-Si BILAYER TRANSMISSION AND REFLECTION DUAL QUARTER- WAVE RETARDER AT $\phi=\mathbf{8 2}^{\circ}$ AND}

\section{$\lambda=10.6 \mu \mathrm{m}$}

As a specific design we take $\left(N_{1}, N_{2}\right)=(1.35,3.42)$, which corresponds to a cryolite-Si bilayer at the $10.6-\mu \mathrm{m}$ $\mathrm{CO}_{2}$-laser wavelength, and $\phi=82^{\circ}$ angle of incidence.

The normalized thickness $\zeta_{1}$ is assigned constant values from 0 to 1 in steps of 0.1 , and for each value of $\zeta_{1}, \zeta_{2}$ is scanned over the full range $0-1$ in finer steps. Figure 4 shows the resulting family of contours of $\rho_{t}$ in the complex plane, as calculated from Eq. (3). These contours are circles because $\rho_{t}$ is a bilinear function of $X_{2}$ for con- 
stant $X_{1}$ according to Eq. (3), and the bilinear transformation maps the unit circle of $X_{2}$ onto a circle ${ }^{9}$ of $\rho_{t}$. Points of intersection of the circles with the imaginary axis in

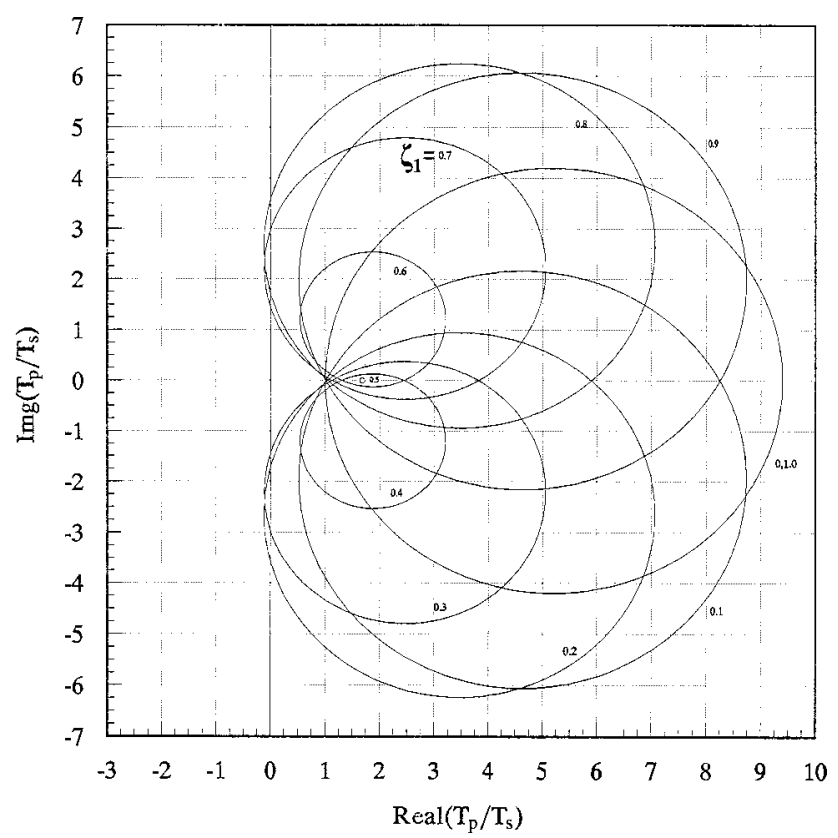

Fig. 4. Family of contours of the ratio of complex-amplitude $p$ and $s$ transmission coefficients $\rho_{t}$ in the complex plane for a cryolite-Si bilayer with refractive indices $(1.35,3.42)$, as calculated from Eq. (3), at $\phi=82^{\circ}$ angle of incidence. The normalized thickness of the first layer, $\zeta_{1}$, assumes constant values marked by each curve while the normalized thickness of the second film, $\zeta_{2}$, is scanned.

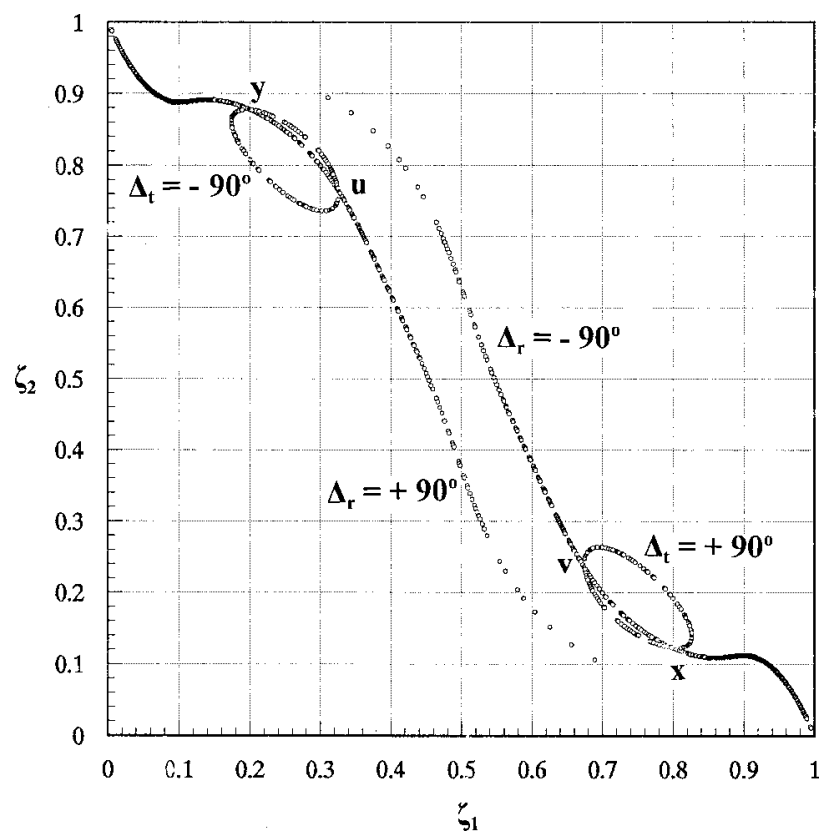

Fig. 5. Multiple solutions $\left(\zeta_{1}, \zeta_{2}\right)$ for bilayer transmission quarter-wave retarders (QWR's), for both $\Delta_{t}=+90^{\circ}$ and $\Delta_{t}$ $=-90^{\circ}$, are presented by the closed contours. Superimposed are the corresponding solution loci for reflection phase shifts $\Delta_{r}$ $=+90^{\circ}$ and $\Delta_{r}=-90^{\circ}$ for the cryolite-Si bilayer at $\phi=82^{\circ}$ angle of incidence. The intersection points $x, y, u$, and $v$ represent bilayers that function as a dual QWR in transmission and reflection.

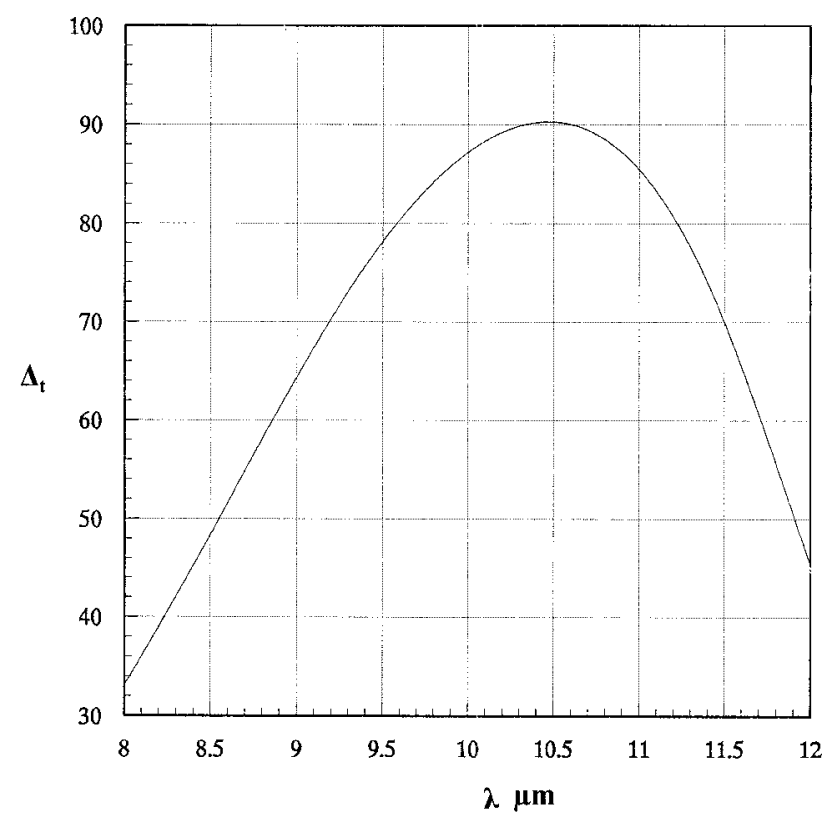

Fig. 6. $\Delta_{t}$ as a function of wavelength around $\lambda=10.6 \mu \mathrm{m}$ for the cryolite-Si pellicle QWR.

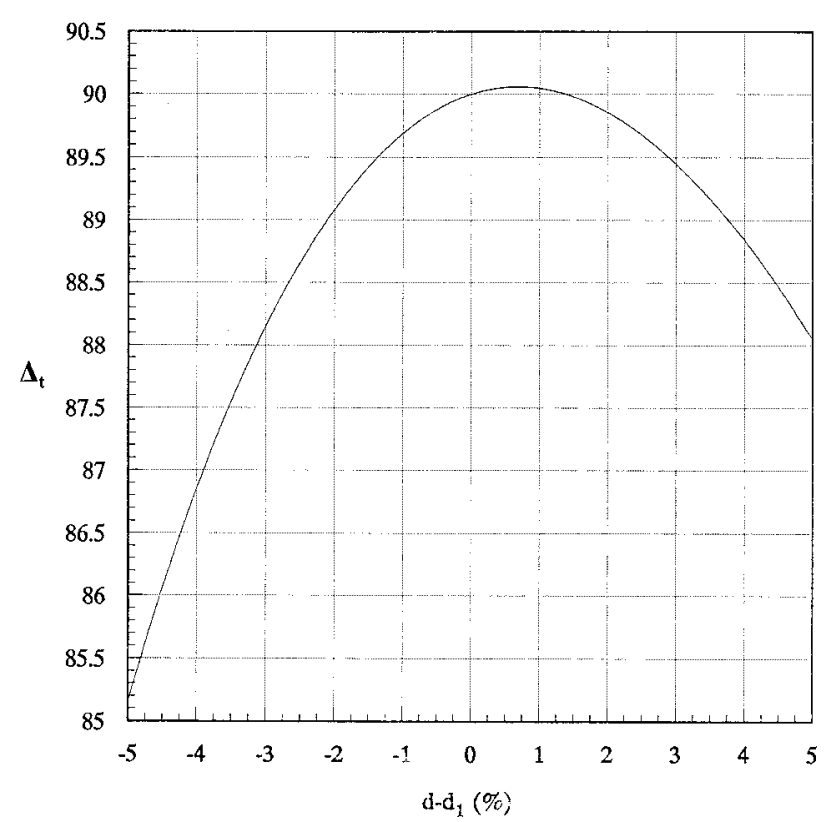

Fig. 7. Effect on $\Delta_{t}$ of errors of $\pm 5 \%$ of the cryolite film thickness $d_{1}$ in the cryolite-Si pellicle QWR.

Fig. 4 correspond to bilayers that produce a QWR in transmission, i.e., $\Delta_{t}= \pm 90^{\circ}$.

Multiple solutions $\left(\zeta_{1}, \zeta_{2}\right)$ for a transmission QWR, for both $\Delta_{t}=+90^{\circ}$ and $\Delta_{t}=-90^{\circ}$, are presented by the closed contours in Fig. 5. Superimposed in Fig. 5 are the corresponding solution loci for reflection phase shifts $\Delta_{r}$ $=+90^{\circ}$ and $\Delta_{r}=-90^{\circ}$ for the same bilayer. A surprise is the intersection of the loci of transmission and reflection QWR's at the four points $x, y, u$, and $v$. These points represent four bilayers that function as a dual QWR $\left(\Delta_{t, r}= \pm 90^{\circ}\right)$ in transmission and reflection.

We further consider the design marked $x$ in Fig. 5 . The normalized and least metric film thicknesses for this 
bilayer are $(0.7992,0.1228)$ and $(4.6164,0.1988) \mu \mathrm{m}$, respectively. If the dispersion of the two film materials is neglected, the effect on $\Delta_{t}$ of shifting the wavelength around the design wavelength $\lambda=10.6 \mu \mathrm{m}$ is shown in Fig. 6. The QWR is reasonably achromatic in the neighborhood of the design wavelength $\left(\left|\delta \Delta_{t}\right|<1^{\circ}\right.$ between $\lambda=10.3$ and $10.7 \mu \mathrm{m}$ ).

Figures 7 and 8 show the effect on $\Delta_{t}$ of errors of $\pm 5 \%$ of the thicknesses $d_{1}$ and $d_{2}$, respectively, at the design wavelength $\lambda=10.6 \mu \mathrm{m}$ and the angle of incidence $\phi=82^{\circ}$. It is apparent that the layer thicknesses should be controlled to within $\pm 2 \%$ to limit the phase error to $<1^{\circ}$.

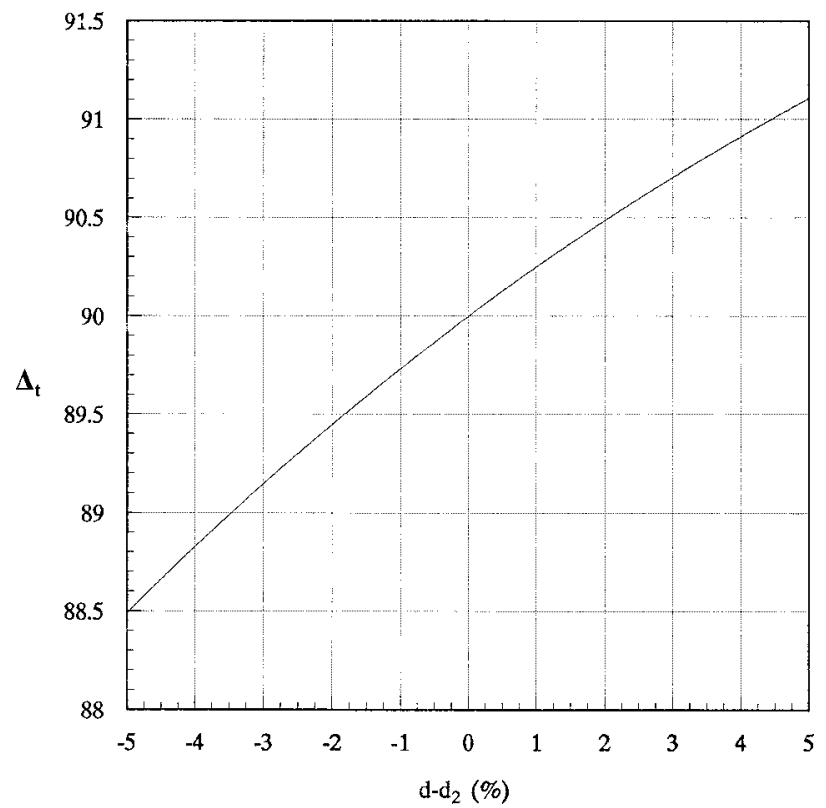

Fig. 8. Effect on $\Delta_{t}$ of errors of $\pm 5 \%$ of the Si film thickness $d_{2}$ in the cryolite-Si pellicle QWR.

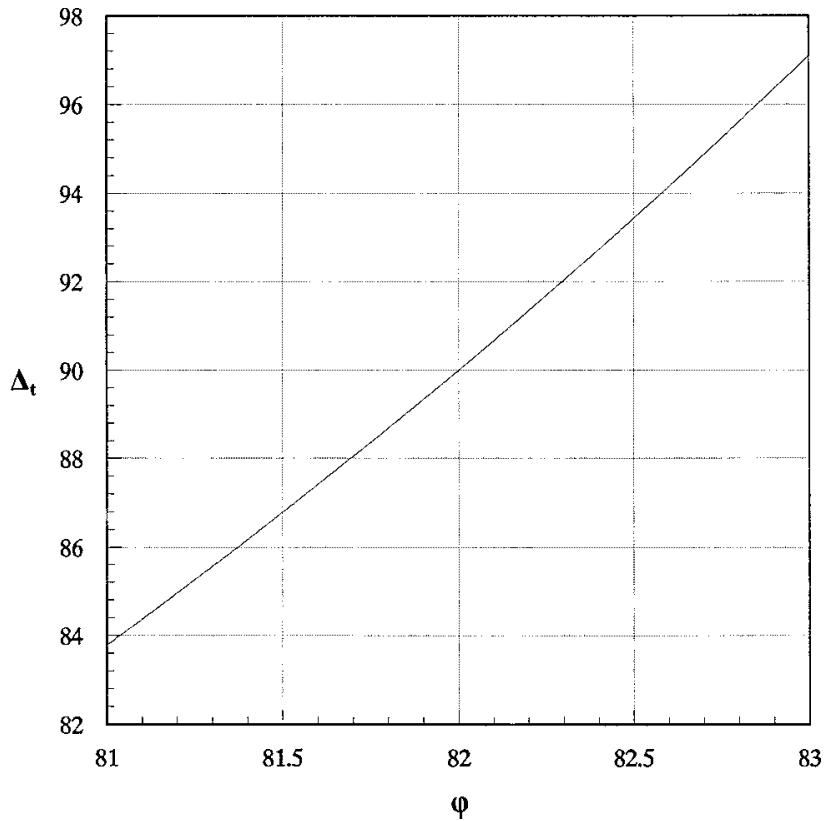

Fig. 9. Effect on $\Delta_{t}$ of shifting the angle of incidence $\phi$ by \pm 1 around $\phi=82^{\circ}$ for the cryolite-Si pellicle QWR.

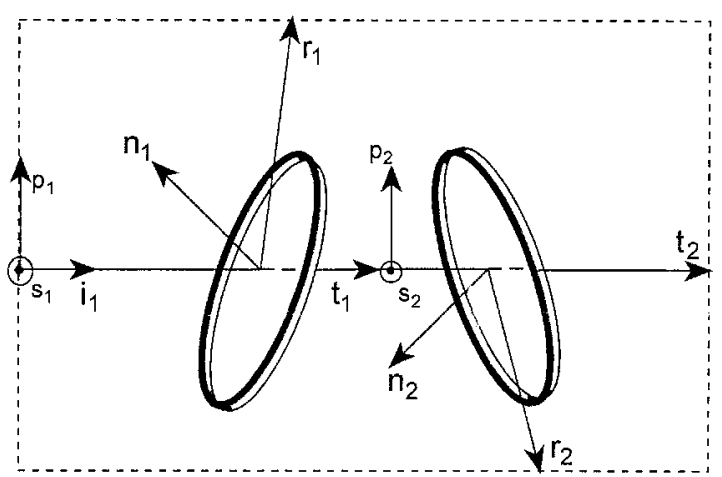

Fig. 10. Two oppositely tilted pellicle eighth-wave retarders in series function as a QWR.

Figure 9 shows the effect on $\Delta_{t}$ of shifting the angle of incidence by $\pm 1^{\circ}$. As may be expected, operation at the large angle $\phi=82^{\circ}$ requires a collimated (e.g., laser) beam with milliradian beam divergence.

Because of the unequal throughput for the $p$ and $s$ polarization (diattenuation ${ }^{10}$ ) of the bilayer QWR, the electric field vector of incident linearly polarized light must be oriented at the appropriate azimuth $\theta$ (measured from the plane of incidence) to produce an output (transmitted) circularly polarized beam. For design $x$ we determine $\theta$ $=59.862^{\circ}$, and the insertion loss for this coated-pellicle circular polarizer under this condition is 0.3395 .

It is possible to design a bilayer EWR without diattenuation. For the same refractive indices $\left(N_{1}, N_{2}\right)$ $=(1.35,3.42)$ and the same angle of incidence $\phi$ $=82^{\circ}$, an EWR is achieved with normalized thicknesses $\left(\zeta_{1}, \zeta_{2}\right)=(0.1009,0.9594)$. In this case $\Delta_{t}=-45^{\circ}$, and the throughputs (intensity transmittances) for the $p$ and $s$ polarizations are the same and equal $84.5 \%$. If two such pellicles are used in series and with equal and opposite tilts (Fig. 10), a QWR with improved field of view is obtained. Other two-pellicle designs are possible, at lower angles of incidence, in which the planes of incidence at the two pellicles are made orthogonal to equalize the $p$ and $s$ net transmittances.

\section{CONCLUSION}

Pellicles are used traditionally as light, yet quite sturdy beam splitters. ${ }^{11}$ In this paper it is shown that, by appropriate design, a bilayer pellicle of optically isotropic materials can introduce $90^{\circ}$ differential phase shift between the $p$ and $s$ linear polarization components in transmission and hence can function as a simple QWR with negligible lateral or angular shift. To lower the operating angle of incidence and remove diattenuation require multilayer-coated pellicles. This extension of the present work falls outside the scope of this paper.

Address correspondence to R. M. A. Azzam at the location on the title page or by e-mail, razzam@uno.edu.

\section{REFERENCES}

1. See, for example, D. Clarke and J. F. Grainger, Polarized Light and Optical Measurement (Pergamon, Oxford, UK, 1971). 
2. D. A. Holmes, "Wave optics theory of rotatory compensators," J. Opt. Soc. Am. 54, 1340-1347 (1964).

3. R. M. A. Azzam, "Transmission ellipsometry on transparent unbacked or embedded thin films with application to soap films in air," Appl. Opt. 30, 2801-2806 (1991).

4. W. H. Southwell, "Multilayer coating design achieving a broadband $90^{\circ}$ phase shift," Appl. Opt. 19, 2688-2692 (1980).

5. J. H. Apfel, "Phase retardance of periodic multilayer mirrors," Appl. Opt. 21, 733-738 (1982).

6. M. M. K. Howlader and R. M. A. Azzam, "Periodic and quasiperiodic nonquarterwave multilayer coatings for 90 deg reflection phase retardance at 45-deg angle of incidence," Opt. Eng. 34, 869-875 (1995).

7. R. M. A. Azzam and N. M. Bashara, Ellipsometry and Polarized Light (North-Holland, Amsterdam, 1987).

8. E. D. Palik, ed., Handbook of Optical Constants of Solids (Academic, New York, 1985).

9. A. Kyrala, Applied Functions of a Complex Variable (WileyInterscience, New York, 1972).

10. R. A. Chipman, "Polarization analysis of optical systems," Opt. Eng. 28, 90-99 (1989).

11. J. A. Dobrowolski, "Optical properties of films and coatings," in Handbook of Optics, M. Bass, editor-in-chief (McGraw-Hill, New York, 1995), Vol. I, Chap. 42. 